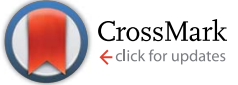

Cite this: Chem. Sci., 2017, 8, 2710

\title{
Highly specific noninvasive photoacoustic and positron emission tomography of brain plaque with functionalized croconium dye labeled by a radiotracer $\dagger$
}

\author{
Yajing Liu, ${ }^{a}$ Yanping Yang, ${ }^{\mathrm{b}}$ Mingjian Sun, ${ }^{\mathrm{c}}$ Mengchao Cui, ${ }^{\mathrm{b}}$ Ying Fu, ${ }^{\mathrm{c}}$ Yu Lin, ${ }^{\mathrm{a}}$ Zijing Li ${ }^{\star a}$ \\ and Liming Nie *a $^{-}$
}

Highly-efficient targeting probes are desirable for disease diagnosis and functional imaging. However, most of the current near-infrared (NIR) probes suffer from low signal conversion, insufficient photostability, poor probe specificity, and limited functions. Herein, an NIR ultrahigh absorbing croconium dye for amyloid (CDA) was designed and synthesized to specifically bind to cerebrovascular amyloid without antibody linkage. This unique CDA is able to strongly bind the hydrophobic channels of amyloid beta (A $\beta$ ) fiber with a very strong binding energy of $-9.3 \mathrm{kcal} \mathrm{mol}^{-1}$. Our experimental results demonstrate that the amphipathic dye with an intense absorption peak at $800 \mathrm{~nm}$ generated a significant local temperature surge under low-power laser irradiation. Compared with representative prominent indocyanine green, Prussian blue, and gold nanorods, this probe can produce the strongest photoacoustic signal based on the same mass concentration. Labeled with radioactive ${ }^{18} \mathrm{~F}$, this multifunctional probe allowed for the ultrasensitive photoacoustic tomography (PAT)/positron emission tomography (PET)/fluorescence imaging of $A \beta$ plaques in the brain cortex. Featured with high spatial resolution and optical specificity, PAT was intrinsically suitable for imaging pathological sites on cortical vessels, whereas PET revealed whole-body anatomy with quantitative biodistribution information. Our study shows that a CDA-based

Received 28th October 2016 Accepted 8th January 2017

DOI: $10.1039 / \mathrm{c} 6 s c 04798 \mathrm{j}$

www.rsc.org/chemicalscience functionalized dye aided with PAT and PET is capable of plaque diagnosis and localization.

\section{Introduction}

Near-infrared (NIR) dyes with a high quantum yield, good biocompatibility, and a fast clearance have gained extensive attention in materials chemistry, optical imaging, and relevant biomedical applications. ${ }^{1-3}$ Because of its easy operation, low cost, and real-time monitoring, near-infrared fluorescence (NIRF) imaging is widely used for detection in various pathophysiological procedures and pre-clinical applications., ${ }^{4,5}$ However, there are few functional NIR dyes that are currently feasible for the detection of specific diseases due to three main weaknesses: (1) poor properties such as suboptimum optical absorption, insufficient stability, poor hydrophilicity, and a low

\footnotetext{
${ }^{a}$ State Key Laboratory of Molecular Vaccinology, Molecular Diagnosis \& Center for Molecular Imaging and Translational Medicine, School of Public Health, Xiamen University, Xiamen 361102, People's Republic of China. E-mail: zijing.li@xmu.edu. cn; nielm@xmu.edu.cn

${ }^{b}$ Key Laboratory of Radiopharmaceuticals, Ministry of Education, College of Chemistry, Beijing Normal University, Beijing 100875, China

${ }^{c}$ Department of Control Science and Engineering, Harbin Institute of Technology, 92 West Dazhi Street, Nan Gang District, Harbin 150001, China

$\dagger$ Electronic supplementary information (ESI) available. See DOI: 10.1039/c6sc04798j
}

quantum yield are still fatal obstacles for imaging biological tissues in vitro and in vivo; ${ }^{6-8}$ (2) most NIR dyes possess extremely low disease targeting and binding capacities, which greatly preclude their use in bioapplications; ${ }^{9}$ (3) there is no rational or ideal approach for developing an NIR dye with complementary imaging capabilities for positron emission tomography (PET)/photoacoustic tomography (PAT) or PAT/ PET/NIR fluorescence on one platform.

Cerebral amyloid angiopathy (CAA) occurs in most patients with Alzheimer's disease (AD), which is characterized by amyloid beta $(A \beta)$ deposition in the walls of leptomeningeal arteries, cortical arteries and veins in the brain. ${ }^{10}$ Considering that $\mathrm{A} \beta$ deposits can occur simultaneously or separately in both blood vessel walls and brain parenchyma, it is critical to develop a precise strategy to map the abnormality for further pinpointing CAA in vivo. ${ }^{11}$ However, the development of effective amyloid-specific optical agents still remains a great challenge. Bacskai et al. reported that vascular amyloid deposits in mice were labeled by thioflavin-T, which, however, has drawbacks of nonspecific binding and a short absorption peak at $500 \mathrm{~nm} \cdot{ }^{12} \mathrm{In}$ addition, there are fewer binding sites associated with the $A \beta$ plaque of the vessels than those reported for the $\mathrm{AD}$ brain. ${ }^{13}$ Therefore, this demands a diagnostic technique suitable for 
vascular imaging with a high sensitive agent that is specific to plaque.

PET, a powerful tool for the early detection of $\mathrm{AD}$, can detect $\mathrm{A} \beta$ deposits with high sensitivity using amyloid specific tracers, enabling in vivo pathological and molecular diagnosis. ${ }^{14}$ However, limited research has been done on the development of imaging agents for the effective diagnosis of CAA. For instance, $\left[{ }^{11} \mathrm{C}\right]$ Pittsburgh compound $\mathrm{B}$ and $\left[{ }^{18} \mathrm{~F}\right]$ florbetapir $\left(\left[{ }^{18} \mathrm{~F}\right] \mathrm{AV}-45\right)$ have shown promise for non-invasive amyloid imaging in $\mathrm{AD}$ patients. ${ }^{15}$ Yet, these two compounds bind to $A \beta$ aggregates in both blood vessels and parenchymal brain tissue. $^{16,17}$ In order to target $A \beta$ located in vasculature, polyethylene glycol modified bivalent and trivalent ligands or a ${ }^{68} \mathrm{Ga} /{ }^{99 \mathrm{~m}} \mathrm{Tc}$ conjugated complex was synthesized either with a larger molecular weight or negative electric charges to limit their permeation of blood vessels. ${ }^{18-20}$ On the downside, these compounds demanded an extra efficient ligand and were only examined in vitro but not in any living subjects. Moreover, PET is impeded by a low spatial resolution and requires an onsite cyclotron along with a radioactive source. In contrast to nuclear imaging, optical imaging offers the benefits of high sensitivity, rapid frame rates, and detection safety.

PAT, a new and emerging technique, has demonstrated prosperous imaging capabilities for samples ranging from organelles to organs, and especially for blood vessels since this method is intrinsically sensitive to hemoglobin. ${ }^{21}$ By converting a pulsed laser into ultrasound, it has opened up a new frontier for bioimaging of the brain with improved spatial resolution and increased penetration depth. ${ }^{22}$ With endogenous contrasts, Yao et al. applied a functional photoacoustic (PA) microscopy system to image the cerebral metabolism and vascular morphology of a mouse brain. ${ }^{23}$ However, the lack of amyloidspecific contrast has confined its use in further disease-oriented applications. Therefore, the combination of using an intrinsic specific probe and noninvasive imaging method is urgent in visualizing the morphology and pathology of cerebral vascular plaque.

In our study, we designed a croconium dye for amyloid (CDA) with limited but sufficient permeability to specifically bind with plaque in vessels (Scheme 1). This probe exhibits very strong absorption in the NIR range and produces a much stronger photoacoustic signal than other prominent materials of the

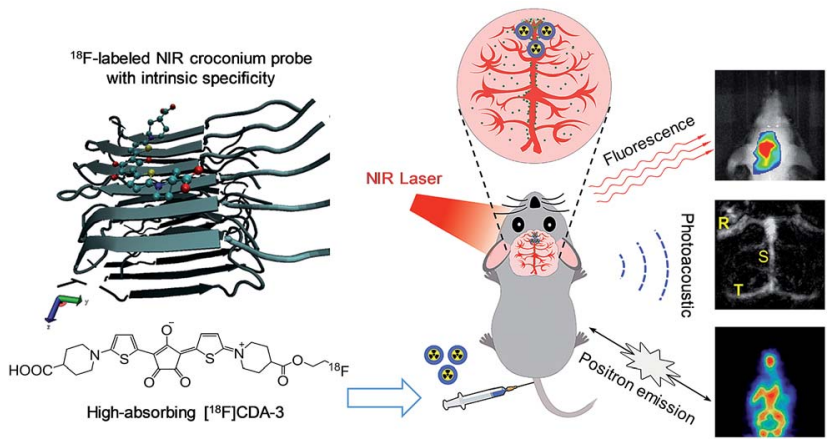

Scheme 1 Schematic illustration of intrinsic targeting $\left.{ }^{18} \mathrm{~F}\right] \mathrm{CDA}-3$ for the ultrasensitive PAT/PET/NIR imaging of brain plaque. same mass concentration. Moreover, the unique material is able to strongly bind the hydrophobic amino acid of the plaque with a strong binding energy, which can significantly improve the detection sensitivity without antibody labeling. After irradiation with an NIR laser, the CDA-3 solution displayed a surging temperature increase from 26 to $63{ }^{\circ} \mathrm{C}$. The hyperthermia that has a great effect on amyloid formation can act as a promising way to dissolve $A \beta$ deposits. ${ }^{24}$

Multimodality imaging that provides both anatomical and functional information has brought a new avenue in precision medical research. ${ }^{25,26}$ To the best of our knowledge, this is the first report on the application of unique croconium dye labeling with an efficient radiotracer for in vivo versatile PAT/PET/NIR fluorescence imaging. As a noninvasive method, PAT provides endogenous absorption contrast from hemoglobin and exogenous molecular contrast from the amyloid specific NIR dye, permitting the real-time visualization and monitoring of cerebral vasculature plaque. Herein, we also report for the first time the evaluation of a specific PAT probe without antibody-labeling for $\mathrm{A} \beta$ plaque targeting imaging in a transgenic $(\mathrm{Tg})$ mouse model.

In addition, our proposed $\left[{ }^{18} \mathrm{~F}\right] \mathrm{CDA}-3$ is among the very few PET probes that can specifically bind plaque in CAA and is available for in vivo whole-body imaging. As expected, significant advantages such as excellent sensitivity, a high spatial resolution and superior quantification are apparent in our NIR fluorescent PAT integrated with PET strategy. Our study may provide a new tool to detect brain plaque at an early stage and unveil the exact mechanisms lying behind neurovascular dysfunctions, for example, the narrowing and rupture of vessels, and related dementia.

\section{Results and discussion}

Inspired by the "aromatic ring-conjugated bridge-aromatic ring" planar backbone structure, we fabricated a series of biocompatible dyes with the croconium or squarylium motif (Fig. 1a and b). The detailed synthesis processes are schematically illustrated in Schemes S1 \& S2. $\dagger$ All of the intermediates, the final CDA and the squarylium dye for the amyloid (SDA) were characterized using ${ }^{1} \mathrm{H}$ nuclear magnetic resonance (NMR), ${ }^{13} \mathrm{C}$ NMR and highresolution mass spectrometry (HRMS) (Fig. S1-S9†). The CDA compounds were further dissolved in an $\mathrm{NaHCO}_{3} / \mathrm{KHCO}_{3}$ solution for subsequent applications.

CDA with an electron donor- $\pi$-acceptor- $\pi$-donor structure exhibits excellent NIR absorption properties (604 $\mathrm{nm}$ for CDA-1, $779 \mathrm{~nm}$ for CDA-2, and $798 \mathrm{~nm}$ for CDA-3). The croconium ring of CDA is a stable annulus mesoionic compound with an electron acceptor heart and an electron donor wing, leading to the strong NIR absorption (Fig. 1c-e). The two thiophene structures are also donor groups, and contribute to the high affinity of $A \beta$ through binding to the hydrophobic channel. The stronger electron withdrawing ability of the croconic acid ring suggests that CDA may exhibit a more bathochromic shift and a higher sensitivity than its squaraine analogues. ${ }^{27}$ In Fig. S10, $\uparrow$ the peak absorption wavelengths of SDA- 1 and SDA- 2 were $491 \mathrm{~nm}$ and $658 \mathrm{~nm}$, which show a $113 \mathrm{~nm}$ and $121 \mathrm{~nm}$ blue-shift for CDA-1 and CDA-2, respectively. 

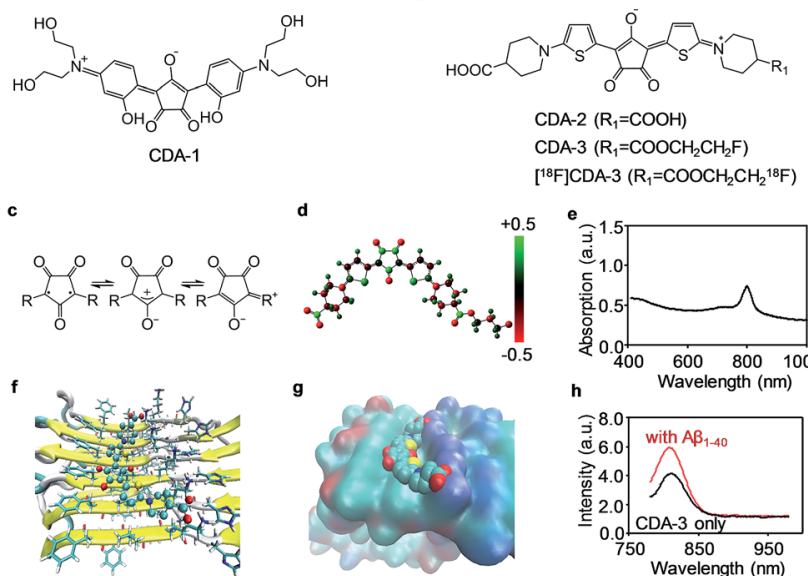

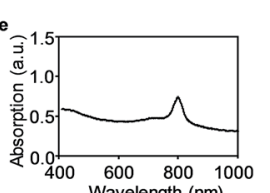

h



Fig. 1 ( $a$ and b) Chemical structures of the probes based on donoracceptor-donor backbones. (c) Resonance structures of a croconate dye: diradical and mesoionic forms. (d) Charge distribution from geometry optimizations of CDA-3 performed at the B3LYP/6-31G (d, p) level in the water phase. (e) UV-Vis absorption curve of CDA-3. ( $f$ ) Molecular docking results of CDA-3 on $A \beta_{1-40}$ fibers. The near-flat geometries of CAD-3 bound to the hydrophobic Val18_Phe20 channel formed by side-chain ladders, with $\mathrm{H}$ bonds formed. (g) The merged docking conformations of CDA-3 on $A \beta$ fibrils presented as transparent material. (h) Fluorescence intensity of CDA-3 and CDA-3 upon interaction with the $A \beta_{1-40}$ aggregates.

We also compared the stability of CDA- 1 and CDA-3 in their aqueous solutions. Fig. S11† illustrates that CDA-2 and CDA-3 had good stability even after 7 days in aqueous solution, whereas CDA-1 tended to aggregate after 1 day. In this work, we aimed to combine NIR fluorescence and the PA imaging technique with the ${ }^{18} \mathrm{~F}$ PET tool to assess the performance of the croconium probe in the CAA disease model. Mono-fluorinated CDA-3 and its radioligand, which were achieved by etherification of CDA-2, were selected for further use (Scheme $\mathrm{S} 2 \dagger$ ). After being incubated with ${ }^{18} \mathrm{~F}^{-}$in aqueous solution, over $30 \%$ of the precursor (complex 10) was radiolabeled for $5 \mathrm{~min}$. By removal of the free ${ }^{18} \mathrm{~F}^{-}$, purified $\left[{ }^{18} \mathrm{~F}\right] \mathrm{CDA}-3$ was obtained with over $85 \%$ yield and kept stable over $2 \mathrm{~h}$ in vitro (Fig. S12 $\dagger$ ). After incubation with human serum albumin (HSA), the absorption spectra of CDA-3 remained almost unchanged at 1, 2, 4, 6 and $8 \mathrm{~h}$ (Fig. S13†). This result illustrated the good biological stability of CDA-3.

Very lipophilic compounds can easily overcome the blood-brain barrier (BBB) but are unable to accurately localize $\mathrm{A} \beta$ deposits between blood vessels and cerebral parenchyma. CAA amyloid aggregates generally originate in the vascular wall, especially in the early stage. Thus, imaging agents targeting amyloid aggregates in CAA should have a certain ability to penetrate limited membranes of the blood vessels. Because of the adequate $\log D$, negative charge, and relatively high molecular weight $(\mathrm{MW}=574)$ near the generally accepted cut-off point, CDA-3 might be an ideal candidate for labelling vascular $A \beta$ depositions, unlike a previously reported croconaine dye. ${ }^{28}$

To further explore the binding mechanism of this newly proposed agent to $A \beta$ fibers, the binding site, thermodynamics

and binding energy were simulated using a molecular docking technique, a powerful tool for studying protein-ligand interactions. ${ }^{29-31}$ As shown in Fig. 1f, the optimized geometry of the CDA compounds was a trapezoid plane with two hydrophilic terminal groups stretching out. The docking result indicated that only one site was located on the symmetrical surfaces of the fibril. CDA-3 is lying above Val18, and parallel and leaning to Lys16 with hydrogen bonds formed between the carboxylate ion of CDA-3 and the carboxyl of lysine (Fig. 1g).

Most of the existing probes share the same site with 6-iodo2-(4-dimethylamino)phenylimidazo[1,2-a]pyridine (IMPY) and nestle into the hydrophobic Val18_Phe20 channel. $^{32}$ The calculated binding energy for CDA-3 was $-9.3 \mathrm{kcal} \mathrm{mol}^{-1}$, which is much more negative compared to $-4.9 \mathrm{kcal} \mathrm{mol}^{-1}$ for IMPY to $A \beta_{1-42}$, and suggests that the CDA compound fits rigorously into the binding pocket. ${ }^{33}$ This Lys16_Val18_Phe20 hydrophilic-hydrophobic dual-channel runs longitudinally to the fibril axis. The channel length and potential binding sites would increase with fibril elongation as more monomers are added. Therefore, it is forcefully confirmed that this binding site is mostly responsible for the binding of CDA-3 to the $\mathrm{A} \beta_{1-40}$ fibrils.

To examine the feasibility of CDA-3 for detecting CAA diseases, we performed in vitro fluorescence staining and in vivo noninvasive NIR and PA imaging. The excitation wavelength and emission wavelength of CDA-3 was $760 \mathrm{~nm}$ and $811 \mathrm{~nm}$, respectively. A very weak interaction was observed in the presence of the $A \beta_{1-40}$ monomers and HSA as shown in Fig. S14. $\dagger$ In contrast, the fluorescence intensity increase for CDA-3 upon binding to the $A \beta_{1-40}$ aggregates suggested an obvious interaction between CDA-3 and the $A \beta_{1-40}$ aggregates (Fig. $1 \mathrm{~h}$ ).

Here, we also evaluated this croconium dye as a novel PA contrast agent for imaging $A \beta$ plaque in the brain. The PA properties of CDA-3 were compared with some of the most commonly used probes such as indocyanine green (ICG), Prussian blue (PB), and gold nanorods (GNRs). The absorption peaks of all of these dyes fall in the NIR window (Fig. 2a). After irradiation with an $808 \mathrm{~nm}$ laser $\left(1.0 \mathrm{~W} \mathrm{~cm}^{-2}, 10\right.$ minutes), the temperature of the CDA-3 solution increased quickly from 26 to $63{ }^{\circ} \mathrm{C}$ and the solution exhibited no measurable change in ultraviolet (UV) absorbance after laser irradiation. Under the same conditions, the GNRs and $\mathrm{PB}$ produced a much smaller temperature increase (from 27 to $40^{\circ} \mathrm{C}$ ) (Fig. $2 \mathrm{~b}$ and c). Most of the cyanine dyes (e.g. ICG) have obvious drawbacks such as photobleaching and the loss of heating effect as time increases (Fig. 2c). ${ }^{34,35}$ In contrast, the dramatic absorption spectral shifts of ICG were not found for CDA at the same time. Our result illustrated that CDA-3 was designed as an ideal organic dye with high performing NIR photothermal properties and photostability. ${ }^{28}$

As an NIR organic dye with an excellent photothermal effect, CDA can efficiently generate PA signals under $800 \mathrm{~nm}$ pulsed laser irradiation (Fig. 2d). Fig. 2f displayed the PA signal dependence of CDA as a function of concentration. In addition, we compared the PA properties of CDA with three prominent representative probes, ICG, PB and GNRs. At the same concentration, CDA provided the highest PA signal intensity 


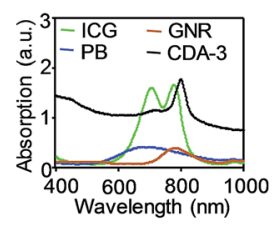

d

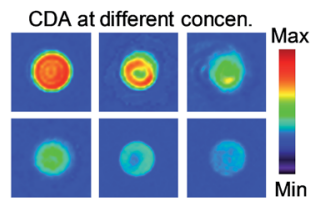

f

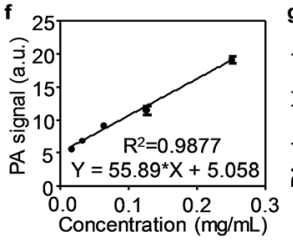

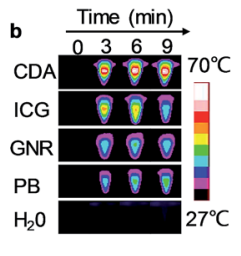
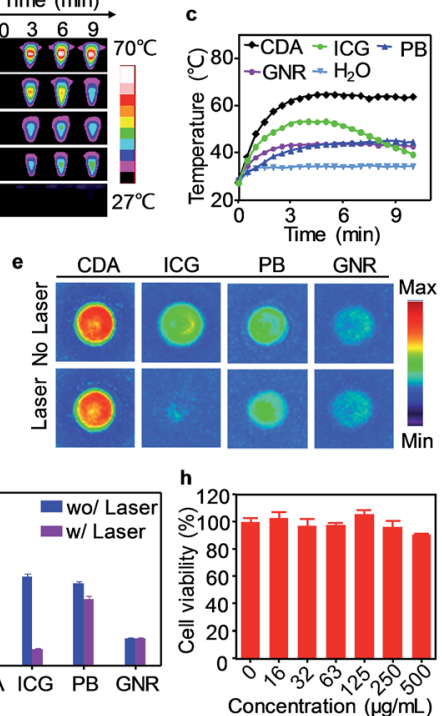

Fig. 2 (a) Absorption spectra obtained for ICG, PB, GNR and CDA-3 in aqueous solution. (b) Photothermal imaging of ICG, PB, GNR, CDA-3 and water under $808 \mathrm{~nm}$ laser irradiation (1 W cm $\mathrm{Cm}^{-2}, 10$ minutes). (c) The corresponding temperature curves from (b). (d) PA imaging of CDA-3 at different concentrations $(1,0.5,0.25,0.125,0.063,0.031$, $0.016,0.008 \mathrm{mg} \mathrm{mL}^{-1}$ ). (e) PA imaging of ICG, PB, GNR and CDA-3 with and without $808 \mathrm{~nm}$ laser irradiation ( $1 \mathrm{~W} \mathrm{~cm}^{-2}, 10$ minutes). (f) PA signal intensity as a function of concentration. (g) PA signal intensities before and after laser irradiation. (h) Cell viability after incubation of CDA-3 at different concentrations with a human neuronal cell line (SH-SY5Y) using MTT assay (each data point was tested based on three replicates).

that was approximately $1.4,1.5$, and 4.5 times higher than that of ICG, PB and GNR, respectively (Fig. 2e). After exposure to a $1.0 \mathrm{~W} \mathrm{~cm} \mathrm{~cm}^{-2}$ laser for $10 \mathrm{~min}$, the PA intensity declines for $\mathrm{PB}$ and GNRs were $18.0 \%$ and $7.3 \%$ respectively. Furthermore, the PA amplitude of ICG dramatically decreased by $\sim 80 \%$, illustrating its susceptibility to laser-induced bleaching (Fig. 2g). Among these four agents, there was a very slight signal decline for CDA (only $\sim 8 \%$ ), revealing its superior potential for longterm imaging. To study the biocompatibility of CDA-3, an MTT (3-(4,5-dimethylthiazol-2-yl)-2,5-diphenyltetrazolium bromide) test was performed using human neuronal cells (SH-SY5Y). Fig. 2h showed that CDA-3 exhibited negligible cytotoxicity even at a relatively high concentration of $0.5 \mathrm{mg} \mathrm{mL}^{-1}$. The acute lethal dose $\left(\mathrm{LD}_{50}\right)$ of CDA-3 via intravenous administration was calculated to be $324.3 \mathrm{mg} \mathrm{kg}{ }^{-1}$ for Kunming mice $(n=8)$, suggesting an extremely low acute toxicity. The SH-SY5Y cells were then incubated with CDA-3 and fluorescence images were acquired using confocal microscopy (FV1200, Olympus, Japan). Fig. S15† illustrated that CDA-3 can be effectively taken up by cells, indicating the high potential application of this probe for biological imaging.

Next, we explored whether CDA-3 specifically labeled $A \beta$ plaque in cerebral blood vessels. The formaldehyde-fixed brain of WT (wild type) and Tg mice was first stained with anti-A $\beta$ antibody (6E10). As shown in Fig. S16, $\uparrow$ the brain sections of $\mathrm{Tg}$ mice were easily subjected to labeling by 6E10. Staining A $\beta$ deposits within cerebral blood vessels and parenchyma was observed on brain sections from the $\mathrm{Tg}$ mice and $\mathrm{AD}$ patient for CDA-3, which was in good agreement with thioflavin-S (Th-S) staining on the same section (Fig. 3a and d). However, there was no apparent labeling in the brain section of WT mice (Fig. S17 $\dagger$ ). To further examine the influence of CDA-3-positive A $\beta$ plaque on blood vessels, staining was performed using a vascular smooth muscle cell (VSMC) marker (phalloidin-Alexa 594) on the paraffin-embedded brain sections. As displayed in Fig. 3b and $\mathrm{c}$, the $\mathrm{A} \beta$ deposits in the blood vessels of $\mathrm{Tg}$ mice were clearly labeled with the VSMC marker. VSMCs were distributed discontinuously in these cerebral vessels, which is a typical hallmark of CAA.

Brain grey and white matter are high scattering and absorbing media and pose a great challenge for deep brain imaging. As an NIR fluorescent dye, the penetration depth of CDA-3 was examined with various brain tissue overlays $(0,1,2,3$ $\mathrm{mm}$ ) (Fig. $4 \mathrm{a}-\mathrm{c}$ ). As the thickness of the brain tissue increased, the fluorescence intensity decreased significantly but still could be detected at the depth of $3 \mathrm{~mm}$. The quantification of the fluorescence intensity is demonstrated in Fig. 4d. This result suggested that CDA-3 could be used as an excellent probe for brain imaging. To detect the biodistribution of CDA-3 dye in vivo, NIRF imaging was performed after an intravenous (i.v.) injection of $0.1 \mathrm{mg} \mathrm{mL}^{-1}$ of CDA-3 was administered to transgenic mice with five familial Alzheimer's disease $(5 \times \mathrm{FAD})$ and littermate WT mice. As can be seen from Fig. 4e and $\mathrm{f}$, there is an obvious difference in brain accumulation between the $5 \times$ FAD and WT groups. At $4 \mathrm{~h}$ postinjection, the $5 \times$ FAD group showed a maximal fluorescence in the brain (Fig. 4g). However, the biodistribution of CDA-3 showed an ignorable brain retention in the WT group, demonstrating that the free dye was quickly eliminated from the body (Fig. 4e). The

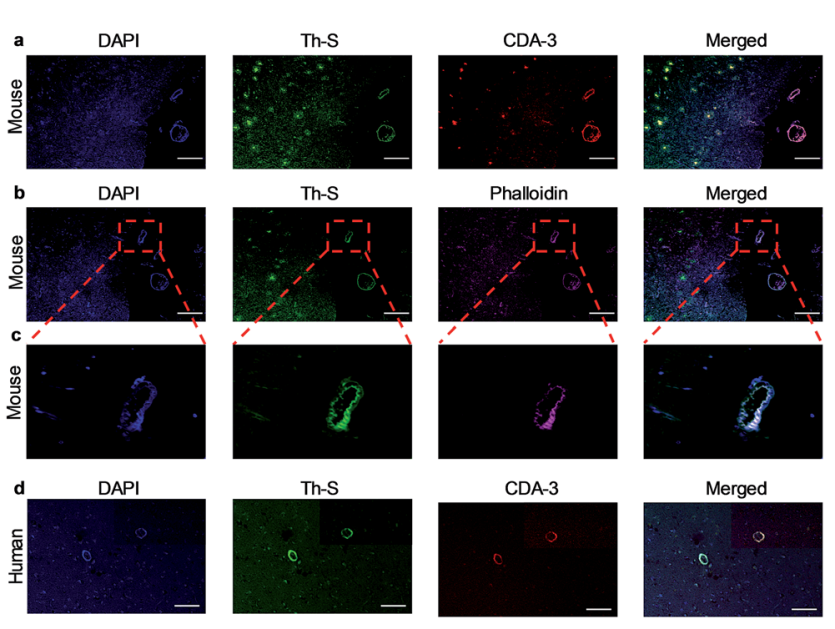

Fig. 3 In vitro fluorescent staining of $\mathrm{A} \beta$ plaque by $\mathrm{CDA}-3$ on brain sections from (a) Tg mice and (d) a human with a filter set of Cy5.5. (b) Vascular smooth muscle cells of Tg mice stained with phalloidin with a filter set of Alexa 594. (c) Higher magnification images show that the VSMC layer of the vessel segment has severe disruption in the VSMC arrangement. The presence and distribution of plaque on the same sections were costained with Th-S (a-d). Scale bars in (a), (b) and (d) are $100 \mu \mathrm{m}$, and in (d) is $50 \mu \mathrm{m}$. 



Fig. 4 Fluorescence imaging of CDA in the brain to examine the penetration depth. (a) White light, (b) fluorescence imaging of CDA-3, (c) fluorescence imaging and (d) the NIRF intensity of CDA-3 embedded in brain tissue with different thicknesses $(0,1,2,3 \mathrm{~mm})$. NIRF images of (e) Tg mice and (f) WT mice at different time points $(0$, $1,4,8 \mathrm{~h})$ after i.v. injection of CDA-3 $\left(0.5 \mathrm{mg} \mathrm{kg}^{-1}\right)$. (g) Relative fluorescence intensities of $\mathrm{Tg}$ and $\mathrm{WT}$ mice quantified at representative time points.

concentration-time curve of CDA-3 in serum shown in Fig. S18† implies its rapid metabolism in living mice. These results suggested that the high fluorescence signal in the brains of the $5 \times$ FAD mice was attributable to the specific binding capacity between the CDA-3 dye and the $\beta$-amyloid plaque.

However, in strongly scattering brain tissue, incident light is mainly attenuated by the cranium, which greatly impedes the application of NIRF imaging for locating the $\beta$-amyloid plaque. Unlike fluorescence imaging, PA imaging can ultrasonically break the strong optical scattering, and simultaneously retain a high penetration depth and spatial resolution. ${ }^{36}$ Owing to the excellent PA performance of CDA-3 in vitro, we then studied the in vivo PA imaging in a CAA model. The PA images of the mice brains are shown in Fig. 5 at different time points after the $\left[{ }^{18} \mathrm{~F}\right]$ CDA-3 injection. From the PA imaging results, it is observed that the functionalized dye achieved better accumulation in the CAA model than in the control group at 1 hour post-injection. After 4 hours from administration, the PA signal of the organic dye displayed a significant improvement in brain blood vessels for the $\mathrm{Tg}$ group, suggesting high $\mathrm{A} \beta$ binding (Fig. 5a). In contrast, the PA signals from the WT group remained almost the same as before (Fig. $5 \mathrm{c}$ ).

To further highlight the brain region, differential PA imaging was performed by subtracting the raw PA images before the agent injection from the PA images at different time points. As shown in Fig. 5b, an outstanding enhanced PA image contrast of the sagittal sinus was observed in the Tg group by comparing the PA signals before and $4 \mathrm{~h}$ after injection. This may be due to the high affinity to $A \beta$ plaque and the gradual accumulation of the dye in brain blood vessels. However, there is no obvious change in the PA signal at different time points for the WT group (Fig. 5d). Quantitative data was also obtained from the same region of interest (ROI) of brain blood vessels. Specifically, the ratio of the PA signals between the Tg and WT mice $[\mathrm{PA}(\mathrm{Tg}) / \mathrm{PA}(\mathrm{WT})]$ reached


$8 \mathrm{~h}$
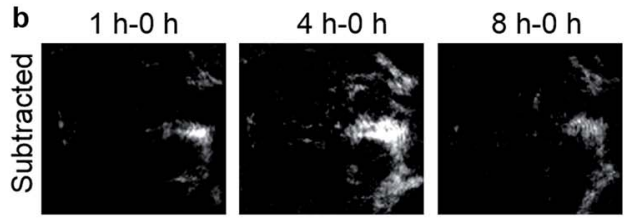

$4 h-8 \mathrm{~h}$

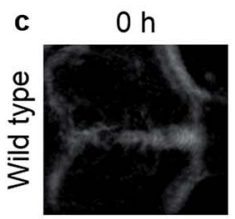

$1 \mathrm{~h}$

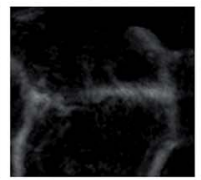

$4 \mathrm{~h}$

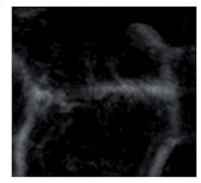

$8 \mathrm{~h}$

d $\quad 1 \mathrm{~h}-\mathrm{Oh}$

$4 \mathrm{~h}-\mathrm{O} \mathrm{h}$

$8 \mathrm{~h}-\mathrm{O} \mathrm{h}$

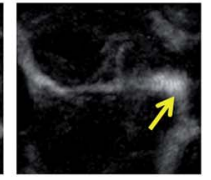

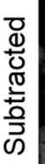


$4 \mathrm{~h}-8 \mathrm{~h}$
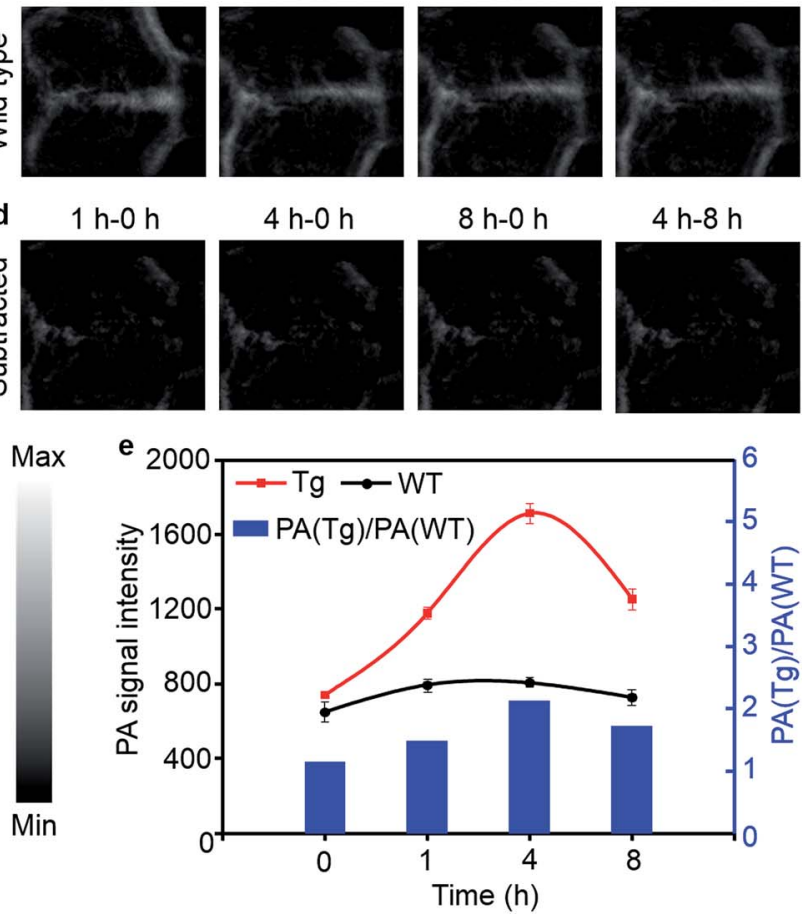

Fig. 5 PA maximum amplitude projection images recorded for (a) $\mathrm{Tg}$ and (c) WT mice after an i.v. injection of $\left[{ }^{18} \mathrm{~F}\right] \mathrm{CDA}-3$ at different time points. PA signal enhancement in mice brains of (b) Tg and (d) WT groups. (e) PA signal intensity of $\left[{ }^{18} \mathrm{~F}\right] \mathrm{CDA}-3$ in the brains of Tg and WT mice (left $Y$ axis) and the values of PA(Tg)/PA(WT) at indicated time points (right $Y$ axis).

2.1 at $4 \mathrm{~h}$, indicative of specific binding by $\mathrm{A} \beta$ plaque (Fig. 5e). The PA signals of brain vessels from the Tg group decreased after $8 \mathrm{~h}$, which contributed to the metabolism of small molecular dyes. Our results demonstrated that the CDA-3 complex showed a powerful capacity to improve the diagnostic value of CAA by the enhanced imaging of plaque.

Although PA imaging is a promising technology, whole-body mapping is still a huge challenge in large animals and humans. As the PET technique is widely used for whole-body imaging in clinics, we explored the ${ }^{18} \mathrm{~F}$ based PET method as a complementary imaging technique. After being analyzed using high performance liquid chromatography (HPLC), we injected a 1.85 million becquerel $(\mathrm{MBq}) / 62.5 \mu \mathrm{g}$ dose of purified $\left[{ }^{18} \mathrm{~F}\right]$ CDA-3 via the tail vein. As can be seen from Fig. 6a, there was a clear distribution of $\left[{ }^{18} \mathrm{~F}\right] \mathrm{CDA}-3$ in the brains of the $\mathrm{Tg}$ mice 

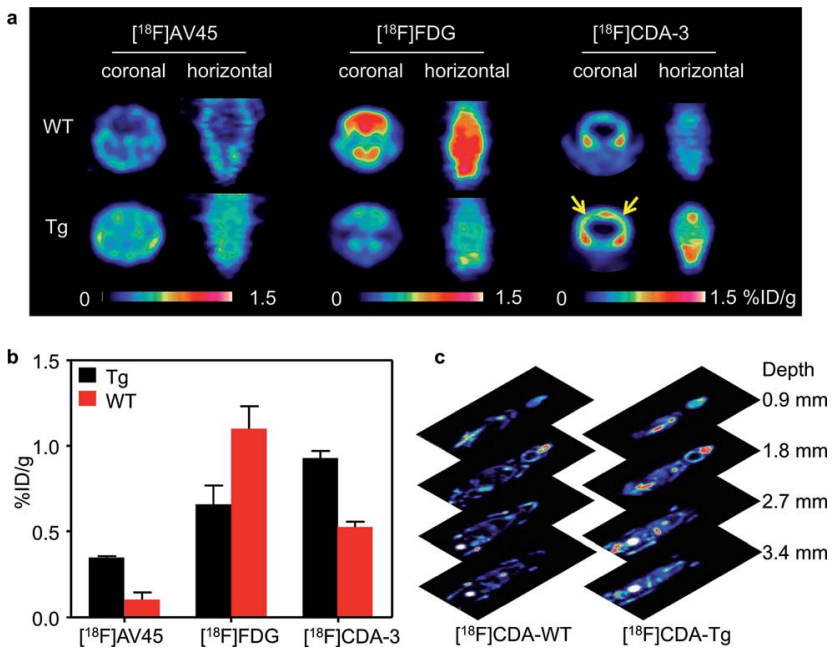

Fig. 6 (a) Multi-tracer PET imaging of mice. Tg mice were ruled out by $\left[{ }^{18} \mathrm{~F}\right] \mathrm{AV}-45$ and $\left[{ }^{18} \mathrm{~F}\right] \mathrm{CDA}-3 \mathrm{PET} / \mathrm{CT}$, indicating $A \beta$ aggregates in the brain. $\left.{ }^{18} \mathrm{~F}\right] \mathrm{FDG}$ PET/CT showed hypometabolism in Tg mice. However, the WT mice were normal without $A \beta$ deposition. (b) Brain uptake of $\left[{ }^{18} \mathrm{~F}\right] \mathrm{CDA}-3$ in the Tg and WT groups. (c) Whole-body PET imaging of $\left[{ }^{18} \mathrm{~F}\right] \mathrm{CDA}-3$ in $\mathrm{Tg}$ and WT mice.

after $1 \mathrm{~h}$. Owing to the lack of $\mathrm{A} \beta$ plaque, there was rarely specific binding in WT mice brains.

To further illustrate this point, study-based analyses for the WT and Tg mice were also carried out using $\left[{ }^{18} \mathrm{~F}\right]$ fluorodeoxyglucose $\left(\left[{ }^{18} \mathrm{~F}\right] \mathrm{FDG}\right)$ and $\left[{ }^{18} \mathrm{~F}\right] \mathrm{AV}-45$. As expected, the $\left[{ }^{18} \mathrm{~F}\right] \mathrm{FDG}$ PET images showed that there was a higher glucose metabolic rate in WT mice than in Tg mice, implying hypometabolism in the brain of AD model. $\left[{ }^{18} \mathrm{~F}\right] \mathrm{AV}-45$, an FDA approved PET probe for $\mathrm{AD}$, also exhibited excellent uptake in the $\mathrm{Tg}$ mice. However, the nonspecific diffusion of the $\left[{ }^{18} \mathrm{~F}\right] \mathrm{AV}-45$ probe in the whole brain hinders its further application for differentiating $A \beta$ plaque between blood vessels and parenchyma. In contrast, owing to the amyloid specific tracer, $\left[{ }^{18} \mathrm{~F}\right] \mathrm{CDA}-3$ PET showed approximately a 2 -fold higher uptake $[0.93 \%$ injection dose per gram (\% ID per g)] in brain blood vessels of the $\mathrm{Tg}$ mice than in the WT group (0.53\% ID per g) (Fig. 6b). Since PET has no tissue penetration limitation, $0.9 \mathrm{~mm}$-thick consecutive coronal slices through the whole mouse were obtained $1 \mathrm{~h}$ after the $\left[{ }^{18} \mathrm{~F}\right] \mathrm{CDA}-3$ injection was administered (Fig. 6c). However, notable defluorination was found for $\left[{ }^{18} \mathrm{~F}\right] \mathrm{CDA}-3$, tomographic imaging of $\mathrm{Tg}$ mice revealed obvious distribution of [18F]CDA-3 in brain in three dimensions, as opposed to low brain accumulation in WT group. Taken together, these results suggest that $\left[{ }^{18} \mathrm{~F}\right] \mathrm{CDA}-3$ is a promising PET probe for the monitoring and early diagnosis of CAA in vivo.

All animal studies were conducted in compliance with the animal care and use guidance established by Xiamen University animal care committee.

\section{Conclusions}

In summary, this is the first study to evaluate the potential of croconium dye as a building block for PA/PET/NIR fluorescence tri-modality imaging for the diagnosis of brain plaque. Depending on the molecular structure, $\left[{ }^{18} \mathrm{~F}\right] \mathrm{CDA}$ has higher selective affinity for cerebrovascular $A \beta$ plaque than neuritic plaque, which does not require an extra antibody or ligand. With its electron donor- $\pi$-acceptor- $\pi$-donor architecture, CDA exhibits excellent NIR absorption and PA response. Under $760 \mathrm{~nm}$ excitation, CDA-3 showed enhanced brain accumulation in the $\mathrm{Tg}$ group where the fluorescence intensity in the brain was significantly improved by more than 4 fold compared with the WT group.

Featuring good photostability and photo-thermal properties, CDA-3 generates a higher PA signal than most of the currently reported PA contrasting nanoagents, such as ICG, PB and GNRs, on a per mass basis. These virtues may permit sensitive PA imaging in living mice at a low systemic injection mass and provide a potential means for the photothermal inhibition of amyloid formation. Moreover, CDA-3 exhibited obvious PA improvement in the cerebral vessel other than parenchyma, which makes it possible for the non-invasive amyloid imaging of CAA. Labeled with a radiotracer, $\left[{ }^{18} \mathrm{~F}\right] \mathrm{CDA}-3$ is feasible for CAA-selective PET imaging in vivo. Compared with conventional $\mathrm{A} \beta$ imaging tracers, $\left[{ }^{18} \mathrm{~F}\right] \mathrm{CDA}-3$ shows great potential for selectively binding CAA deposits in the brain.

The innovation of this study is to explore the potentials of croconium dye as a PET/PA/NIR fluorescent tri-modality imaging ligand for selective affinity towards brain plaque. With enhanced NIR absorption, effective photothermal property, sensitive PA response, and whole-body PET imaging, CDA-3 plays an important role in monitoring cerebrovascular amyloid and will provide powerful support to develop effective prevention and treatment strategies for CAA and other cerebral blood vessel disorders. By replacing croconic acid, ${ }^{18} \mathrm{~F}$, and thiophene with other functional groups, this unique building block will shed light on investigating the underlying mechanisms of brain disorders.

\section{Acknowledgements}

This work was supported by the National Science Foundation of China (81571744, 81501534, and 81601489), National Basic Research Program of China (863 Program 2015AA020502), National Key Basic Research Program of China (2014CB744503), and Science Foundation of Fujian Province (2014Y2004). We thank Prof. Huabei Zhang from Beijing Normal University for assistance with the molecular docking modeling.

\section{References}

1 L. Yuan, W. Lin, K. Zheng, L. He and W. Huang, Chem. Soc. Rev., 2013, 42, 622-661.

2 G. Qian and Z. Y. Wang, Adv. Mater., 2012, 24, 1582-1588.

3 H. Lu, J. Mack, Y. Yang and Z. Shen, Chem. Soc. Rev., 2014, 43, 4778-4823.

4 P. Anees, J. Joseph, S. Sreejith, N. V. Menon, Y. Kang, W. K. Yu, A. Ajayaghosh and Y. Zhao, Chem. Sci., 2016, 7, 4110-4116.

5 X. Wang, J. Sun, W. Zhang, X. Ma, J. Lv and B. Tang, Chem. Sci., 2013, 4, 2551-2556. 
6 Q. Fan, K. Cheng, Z. Yang, R. Zhang, M. Yang, X. Hu, X. Ma, L. Bu, X. Lu, X. Xiong, W. Huang, H. Zhao and Z. Cheng, Adv. Mater., 2015, 27, 843-847.

7 Z. Zha, X. Yue, Q. Ren and Z. Dai, Adv. Mater., 2013, 25, 777782.

8 S. Shi, Y. Liu, Y. Chen, Z. Zhang, Y. Ding, Z. Wu, J. Yin and L. Nie, Theranostics, 2016, 6, 2170-2182.

9 E. M. Sevick-Muraca, Annu. Rev. Med., 2012, 63, 217-231.

10 K. A. Jellinger, Neurodegener. Dis., 2010, 7, 112-115.

11 A. R. Sarkar, Chem. Sci., 2016, 7, 4600-4606.

12 B. J. Bacskai, G. A. Hickey, J. Skoch, S. T. Kajdasz, Y. Wang, G. F. Huang, C. A. Mathis, W. E. Klunk and B. T. Hyman, Proc. Natl. Acad. Sci. U. S. A., 2003, 100, 12462-12467.

13 E. E. Smith and S. M. Greenberg, Stroke, 2009, 40, 2601-2606. 14 B. H. Yousefi, A. Manook, T. Grimmer, T. Arzberger, B. von Reutern, G. Henriksen, A. Drzezga, S. Forster, M. Schwaiger and H. J. Wester, ACS Chem. Neurosci., 2015, 6, 428-437.

15 G. Poisnel, M. Dhilly, O. Moustie, J. Delamare, A. Abbas, D. Guilloteau and L. Barre, Neurobiol. Aging, 2012, 33, 2561-2571.

16 J. V. Ly, G. A. Donnan, V. L. Villemagne, J. A. Zavala, H. Ma, G. O'Keefe, S. J. Gong, R. M. Gunawan, T. Saunder, U. Ackerman, H. Tochon-Danguy, L. Churilov, T. G. Phan and C. C. Rowe, Neurology, 2010, 74, 487-493.

17 M. Carmona-Iragui, A. Fernandez-Arcos, D. Alcolea, F. Piazza, E. Morenas-Rodriguez, S. Anton-Aguirre, I. Sala, J. Clarimon, O. Dols-Icardo, V. Camacho, F. Sampedro, J. Munuera, F. Nunez-Marin, A. Lleo, J. Fortea, B. GomezAnson and R. Blesa, J. Alzheimer's Dis., 2015, 50, 1-7.

18 Z. Zha, S. R. Choi, K. Ploessl, B. P. Lieberman, W. Qu, F. Hefti, M. Mintun, D. Skovronsky and H. F. Kung, J. Med. Chem., 2011, 54, 8085-8098.

19 Z. Zha, J. Song, S. R. Choi, Z. Wu, K. Ploessl, M. Smith and H. Kung, Bioconjugate Chem., 2016, 27, 1314-1323.

20 J. Jia, M. Cui, J. Dai and B. Liu, Mol. Pharm., 2015, 12, 29372946.
21 Y. Liu, L. Nie and X. Chen, Trends Biotechnol., 2016, 34, 420433.

22 Y. Liu, N. Kang, J. Lv, Z. Zhou, Q. Zhao, L. Ma, Z. Chen, L. Ren and L. Nie, Adv. Mater., 2016, 28, 6411-6419.

23 J. Yao, L. Wang, J. M. Yang, K. I. Maslov, T. T. Wong, L. Li, C. H. Huang, J. Zou and L. V. Wang, Nat. Methods, 2015, 12, 407-410.

24 M. Li, X. Yang, J. Ren, K. Qu and X. Qu, Adv. Mater., 2012, 24, 1722-1728.

25 E. Huynh, B. Y. Leung, B. L. Helfield, M. Shakiba, J. A. Gandier, C. S. Jin, E. R. Master, B. C. Wilson, D. E. Goertz and G. Zheng, Nat. Nanotechnol., 2015, 10, 325-332.

26 Y. Zhang, M. Jeon, L. J. Rich, H. Hong, J. Geng, Y. Zhang, S. Shi, T. E. Barnhart, P. Alexandridis, J. D. Huizinga, M. Seshadri, W. Cai, C. Kim and J. F. Lovell, Nat. Nanotechnol., 2014, 9, 631-638.

27 Z. Xiang, E. E. Nesterov, J. Skoch, T. Lin, B. T. Hyman, T. M. Swager, B. J. Bacskai and S. A. Reeves, J. Histochem. Cytochem., 2005, 53, 1511-1516.

28 G. T. Spence, G. V. Hartland and B. D. Smith, Chem. Sci., 2013, 4, 4240-4244.

29 Z. Wang, H. Sun, X. Yao, D. Li, L. Xu, Y. Li, S. Tian and T. Hou, Phys. Chem. Chem. Phys., 2016, 18, 12964-12975.

30 W. J. Hehre, R. Ditchfield and J. A. Pople, J. Chem. Phys., 1972, 56, 2257-2261.

31 A. T. Petkova, W. M. Yau and R. Tycko, Biochemistry, 2006, 45, 498-512.

32 Y. Yang, X. Zhang, M. Cui, J. Zhang, Z. Guo, Y. Li, X. Zhang, J. Dai and B. Liu, Sci. Rep., 2015, 5, 12084.

33 Y. Yang, M. Cui, X. Zhang, J. Dai, Z. Zhang, C. Lin, Y. Guo and B. Liu, J. Med. Chem., 2014, 57, 6030-6042.

34 X. Wu, X. Sun, Z. Guo, J. Tang, Y. Shen, T. D. James, H. Tian and W. Zhu, J. Am. Chem. Soc., 2014, 136, 3579-3588.

35 S. Guha, G. K. Shaw, T. M. Mitcham, R. R. Bouchard and B. D. Smith, Chem. Commun., 2016, 52, 120-123.

36 L. Nie and X. Chen, Chem. Soc. Rev., 2014, 43, 7132-7170. 